\title{
Coherent states for Hopf algebras
}

\author{
Zoran Škoda (zskoda@irb.hr) \\ Theoretical Physics Division, Institute Rudjer Bošković, P.O.Box 180, \\ HR-10002 Zagreb
}

\begin{abstract}
Families of Perelomov coherent states are defined axiomatically in the context of unitary representations of Hopf algebras. A global geometric picture involving locally trivial noncommutative fibre bundles is involved in the construction. If, in addition, the Hopf algebra has a left Haar integral, then a formula for noncommutative resolution of identity in terms of the family of coherent states holds. Examples come from quantum groups.
\end{abstract}

Keywords: coherent states, Hopf algebra, comodule algebra, Ore localization, localized coinvariants, line bundle, resolution of unity

AMS classification: 14A22,16W30,14L30,58B32

Coherent states were at first defined by ScHRÖDINGER in quantum optics, and later extended by many people in various frameworks and generalities; often in the context of complex Lie groups and their real forms ([2, 19]). PERELOMOV ([28, 29]) starts with a real Lie group $G$, and a unitary irreducible representation $T: G \rightarrow$ Aut $V$ on a complex Hilbert space $V$. Fix a vector $v_{0}$ in $V$ so that Lie subgroup $H \subset G$ is its projective isotropy subgroup (i.e. $h \in H$ iff $h v_{0}$ equals $v_{0}$ up to a constant phase). Hence, there is a unitary character $\chi: H \rightarrow S^{1}$ such that $h v_{0}=\chi(h) v_{0}$ for each $h \in H$. For $G$ compact, the representation $T$ extends to a representation of the complexification $G^{\mathbb{C}}$ of $G$.

A family of Perelomov coherent vectors in $V$ is a family of vectors $\{C(u), u \in G / H\}$, such that $C([g])=T(g) v_{0}$ up to a phase. Coherent states are projective classes (rays) of coherent vectors, but in practice one often says "coherent states" for both notions. If $V$ is constructed by the method of geometric quantization, i.e. as the space of holomorphic sections $\Gamma L$ of the corresponding quantization line bundle $L$ over $G / H$, then the coherent vectors may be defined invariantly in terms of that line bundle (30]). For $G$ a compact form of a semisimple Lie group $G^{\mathbb{C}}$, the details are in Section 4 below.

Hopf algebras appear in physics as symmetries of noncommutative and quantum spaces (22, 24, 25, 26, 44]). Algebra $\mathcal{O}(G)=\Gamma \mathcal{O}_{G}$ of regular functions on affine algebraic group $G$ are commutative examples of Hopf algebras (14]) with coproduct $\Delta: \mathcal{O}(G) \rightarrow \mathcal{O}(G) \otimes \mathcal{O}(G) \cong$ $\mathcal{O}(G \times G)$ given by $(\Delta f)\left(g_{1}, g_{2}\right)=f\left(g_{1} \cdot g_{2}\right)$. In fact, the category of commutative Hopf algebras is antiequivalent to the category of affine group schemes ([14, 34]). Hence, the noncommutative Hopf algebras are

(C) 2018 Kluwer Academic Publishers. Printed in the Netherlands.

cohzoki4.tex; 25/10/2018; 8:42; p.1 
thought of as (duals to) noncommutative affine group schemes ([10, 34]; drawback: $\otimes$ is not a categorical product of noncommutative rings). Actions of affine group schemes generalize then to the coactions of Hopf algebras, which can furthermore be "structure groups" of noncommutative fibre bundles. The total space of such a bundle is either a single algebra (affine case) or a more complicated system of algebras or categories with gluing or localizing mechanism to pass between global and local description. Noncommutative fibre bundles with coacting Hopf algebras playing the role of a structure group first appeared in now classical work on smash products and Hopf-Galois extensions.

Then, H-J. SCHNEIDER introduced in [35] a crucial descent theorem supporting the geometric torsor intuition for faithfully flat Hopf-Galois extensions. In a study of noncommutative algebras equipped with differential calculi, MAJID and BRZEZIŃSKI ([7]) discovered a remarkable condition on differential calculi which enter the definition of principal bundles in that case. The coherent states on noncommutative projective homogeneous spaces, exhibited in the present work, seem to need a bundle theory extended in a different direction. To this aim, the present author has extended the concepts of Zariski locally trivial principal fibre bundles (36, 37, 40, and 41, Part I) to the setup where both total and base space are noncommutative (described locally by noncommutative algebras) and not necessarily affine.

Every complex semisimple Lie group $G^{\mathbb{C}}$ is an affine algebraic $\mathbb{C}$ group, and $G^{\mathbb{C}} \rightarrow G^{\mathbb{C}} / B$ is an algebraic principal fibration Zariski locally trivialized in a cover by shifts by action of Weyl group $W$ of the main Bruhat cell (14]). Noncommutative analogues of such fibrations, derived from quantum matrix groups $\mathcal{G}_{q}$, are recently exhibited [36, 37. The fibrations trivialize in coaction-compatible Ore localizations $S_{w}^{-1} \mathcal{G}_{q}$ labeled by the elements $w$ of the Weyl group $W$. The trivializations are explicitly computed using an elaborate Ansatz involving $q$-w-Gauss decompositions (37, Theorems 9-12; proofs in 36] and 41, II).

In noncommutative case, it is not appropriate to seek for individual coherent vectors or rays in representation space $V$. A family of coherent vectors $C$ should be a section of a noncommutative bundle $V \otimes L_{\chi}$ over a noncommutative "coset" space $X$ "parametrizing would-be individual" coherent states, where the fiber $V=V_{\chi}=\Gamma L_{\chi}=\operatorname{Ind}_{\mathcal{B}} \mathbb{C}_{\chi}$ is an analogue of a holomorphically induced representation space, $\chi$ is an analogue of a character of the inducing subgroup $B$ and $L_{\chi}$ is an analogue of the Borel-Weil line bundle. Our noncommutative coset spaces are patched from charts. Local descriptions of $X$ and $C$ in different covers by charts are naturally equivalent. Earlier studies of coherent states for quantum groups ([17, 33]) used computations in a single local chart. One of our goals was to show that states locally computed 
in (33) may be defined a priori, regardless coordinate choices. The main goal was to find a resolution of unity in terms of coherent states of compact quantum groups.

Notation for Hopf algebras: unit map $\eta$, counit $\epsilon$, multiplication $\mu$, coproduct $\Delta$, antipode $S$ (do not confuse with $S$ and $T$ sometimes used for generic Ore subsets in a ring). We use SweEdLER's notation: for coproduct $\Delta(a)=\sum a_{(1)} \otimes a_{(2)}$; for (say right) coactions $\rho(v)=\sum v_{(0)} \otimes v_{(1)}$; and their multiplace extensions (25]). Ground ring $\mathbf{k}$ is any commutative unital (in Sections 1-3, later $\mathbf{k}=\mathbb{C}$ ); the category of left $\mathcal{E}$-modules for a $\mathbf{k}$-algebra $\mathcal{E}$ is denoted $\mathcal{E}_{\mathcal{M}} \mathcal{M}$. The $\mathcal{B}$ comodule analogue has a superscript $\left({ }^{\mathcal{B}} \mathcal{M}\right)$. The right-hand versions have a right sub/super-script instead (e.g. $\mathcal{M}^{\mathcal{B}}$ ), and for bi(co)modules we use combinations.

\section{Prerequisites on Ore localization and covers}

Let $\mathcal{E}$ be an associative unital ring. For any multiplicative subset $S \subset$ $\mathcal{E}$, define category $\mathcal{C}_{l}=\mathcal{C}_{l}(\mathcal{E}, S)$ as follows. Objects of $\mathcal{C}_{l}$ are pairs $(i, Y)$ where $Y$ is a ring and $i: \mathcal{E} \rightarrow Y$ a unital ring homomorphism, such that (i) for each $s \in S$, the image $i(s)$ is invertible; (ii) the set $\left\{i(s)^{-1} i(r) \mid s \in S, r \in \mathcal{E}\right\}$ is a subring of $Y$; (iii) and $i(r)=0$ iff $s r=0$ for some $s \in S$. A morphism $h:(i, Y) \rightarrow\left(i^{\prime}, Y^{\prime}\right)$ is a ring map $h: Y \rightarrow Y^{\prime}$ such that $h \circ i=i^{\prime}$. The left ORE localization of $\mathcal{E}$ at $S$ is a universal object $\left(\iota_{\mathcal{E}}, S^{-1} \mathcal{E}\right)$ in $\mathcal{C}_{l}(\mathcal{E}, S)$. It exists iff $S$ is a left Ore set $(\underline{37}, 39)$.

We denote by $\mathcal{E}$-Mod the Abelian category of left $\mathcal{E}$-modules. Every Ore localization $\left(\iota_{\mathcal{E}}, S^{-1} \mathcal{E}\right)$ induces an exact localization functor by

$$
Q^{*}=Q_{S}^{*}: \mathcal{E}-\operatorname{Mod} \rightarrow S^{-1} \mathcal{E}-\operatorname{Mod}, \quad M \mapsto S^{-1} \mathcal{E} \otimes_{\mathcal{E}} M .
$$

As an example of a localization functor, $Q^{*}$ has a fully faithful right adjoint $Q_{*}$, which is in Ore case exact, equals the restriction of scalars and has its own right adjoint $Q^{!}$. The adjunction morphism $\iota: \operatorname{Id}_{\mathcal{E}-\operatorname{Mod}} \rightarrow$ $Q_{*} Q^{*}$ is given by $\iota_{M}=\iota_{\mathcal{E}} \otimes_{\mathcal{E}} \operatorname{id}_{M}: \mathcal{E} \otimes_{\mathcal{E}} M \cong M \rightarrow Q_{*} Q^{*}(M)$. Denote the composition $Q_{*} \circ Q^{*}=: Q$. It is an endofunctor in $\mathcal{E}$ - Mod. One often denotes $Q_{S}(M)$ and $Q_{S}^{*}(M)$ by $S^{-1} M$.

If $S_{1}$ and $S_{2}$ are two left Ore sets, then the set $S_{1} S_{2}$ of all products $s_{1} s_{2}$, where $s_{1} \in S$ and $s_{2} \in S$, is not necessarily multiplicative, but set $S=S_{1} \vee S_{2}$ multiplicatively generated by the union $S_{1} \cup S_{2}$ is left Ore. Hence the "double" localization $Q_{S_{1} \vee S_{2}}$ is well-defined and $Q_{S_{1} \vee S_{2}}(R)$ has a canonical structure of a ring. Two 'consecutive localizations' (compositions of functors) $Q_{S_{1}} \circ Q_{S_{2}}$ and $Q_{S_{2}} \circ Q_{S_{1}}$, are not rings in general, but they play the role in gluing (see below). Canonical natural 
transformations $Q_{S_{1}} \circ Q_{S_{2}} \rightarrow Q_{S_{1} \vee S_{2}}$ and $Q_{S_{2}} \circ Q_{S_{1}} \rightarrow Q_{S_{1} \vee S_{2}}$ are in noncommutative case rarely isomorphisms ('mutual compatibility' of $Q_{S_{2}}$ with $Q_{S_{1}}$ ), and $Q_{S_{1}} \circ Q_{S_{2}} \neq Q_{S_{2}} \circ Q_{S_{1}}$, so one should not count on this. Note also the natural maps $Q_{S_{1}}\left(\iota_{M}\right): Q_{S_{1}}(M) \rightarrow Q_{S_{1}} Q_{S_{2}}(M)$ and $\iota_{S_{2}^{-1} M}: Q_{S_{2}}(M) \rightarrow Q_{S_{1}} Q_{S_{2}}(M)$.

For geometrical purposes, one considers families of Ore localizations $\left\{\left(\iota_{\lambda}, S_{\lambda}^{-1} \mathcal{E}\right)\right\}_{\lambda \in \Lambda}$ with localization functors $Q_{\lambda}^{*}, Q_{\lambda}$. We abbreviate

$$
\begin{array}{lrl}
\mathcal{E}_{\lambda}:=S_{\lambda}^{-1} \mathcal{E}, & \mathcal{M}_{\mu \nu \ldots \lambda}:=S_{\lambda}^{-1} \ldots S_{\nu}^{-1} S_{\mu}^{-1} M, \\
\iota_{\mu \nu}^{\mu}:=\iota_{\mathcal{E}}: \mathcal{E}_{\mu} \rightarrow \mathcal{E}_{\mu \nu}, & \iota_{\mu \nu M}^{\mu}:=\iota_{\mu \nu}^{\mu} \otimes_{\mathcal{E}} \operatorname{id}_{M}, \\
\iota_{\mu \nu}^{\nu}:=Q_{\nu}\left(\iota_{\mu}\right): \mathcal{E}_{\nu} \rightarrow \mathcal{E}_{\mu \nu}, & \iota_{\mu \nu M}^{\nu}:=\iota_{\mu \nu}^{\nu} \otimes_{\mathcal{E}} \operatorname{id}_{M} .
\end{array}
$$

A family of left (right work as well) Ore localizations $\left\{S_{\lambda}^{-1} \mathcal{E}\right\}_{\lambda i n \Lambda}$ (cf. 31, 37, 40, ), covers $\mathcal{E}$ if the fork diagram

$$
\mathcal{E} \stackrel{\prod_{\lambda} \iota_{\lambda}}{\longrightarrow} \prod_{\lambda \in \Lambda} S_{\lambda}^{-1} \mathcal{E} \stackrel{\stackrel{i_{1}}{\longrightarrow}}{\longrightarrow} \prod_{(\mu, \nu) \in \Lambda \times \Lambda} S_{\nu}^{-1} S_{\mu}^{-1} \mathcal{E}
$$

is an equalizer diagram. The upper right map $i_{1}$ is $\prod_{\lambda} e^{\lambda} \mapsto \prod_{\mu \nu} \iota_{\mu \nu}^{\mu}\left(e^{\mu}\right)$ and the lower right map $i_{2}$ is $\prod_{\lambda} e^{\lambda} \mapsto \prod_{\mu \nu} \iota_{\mu \nu}^{\nu}\left(e^{\nu}\right)$.

When this covering condition holds the analogous equalizer property extends to other $\mathcal{E}$-modules (not only $M=\mathcal{E}$ ):

Globalization lemma. (in wider generality, 31 p. 103) Suppose a finite family of Ore localizations $\left\{S_{\lambda}^{-1} \mathcal{E}\right\}_{\lambda \in \Lambda}$ covers $\mathcal{E}$. Then for every left $\mathcal{E}$-module $M$ the sequence

$$
0 \rightarrow M \rightarrow \prod_{\lambda \in \Lambda} S_{\lambda}^{-1} M \rightarrow \prod_{(\mu, \nu) \in \Lambda \times \Lambda} S_{\mu}^{-1} S_{\nu}^{-1} M
$$

is exact, where the first morphism is $m \mapsto \prod \iota_{\lambda, M}(m)$ and the second is

$$
\prod_{\lambda} m_{\lambda} \mapsto \prod_{(\mu, \nu)}\left(\iota_{\mu, \nu, M}^{\mu}\left(m_{\mu}\right)-\iota_{\mu, \nu, M}^{\nu}\left(m_{\nu}\right)\right) .
$$

Here the order matters: pairs with $\mu=\nu$ may be (trivially) skipped, but unlike in the commutative case we can not confine to the pairs of indices with $\mu<\nu$ only.

\section{Quantum principal bundles using Ore localizations}

Let $\mathcal{B}$ be a Hopf algebra. An algebra $\mathcal{E}$ is a $\mathcal{B}$-comodule algebra if it is given with a $\mathcal{B}$-coaction $\rho$ which is an algebra map ([22, 25, 27]]). For 
commutative $\mathcal{E}$ and $\mathcal{B}$ this means that the affine scheme $E=\operatorname{Spec} \mathcal{E}$ is given a regular action of an affine algebraic group $B=\operatorname{Spec} \mathcal{B}$. An Ore localization $S^{-1} \mathcal{E}$ is $\rho$-compatible (37) if there is a (unique) $\mathcal{B}$ coaction $\rho_{S}$ on $S^{-1} \mathcal{E}$, making $S^{-1} \mathcal{E}$ a $\mathcal{B}$-comodule algebra such that the localization map $\iota_{\mathcal{E}}: \mathcal{E} \rightarrow S^{-1} \mathcal{E}$ is a map of $\mathcal{B}$-comodule algebras. In commutative case, this means that $\operatorname{Spec} S^{-1} \mathcal{E}$ is a $B$-invariant Zariski open subscheme of $\operatorname{Spec} \mathcal{E}$. Localized coinvariants are those $e$ in $S^{-1} \mathcal{E}$ for which $\rho_{S}(e)=e \otimes 1$ i.e. the coinvariants for the "localized" coaction $\rho_{S}$. They form subalgebra $\left(S^{-1} \mathcal{E}\right)^{\text {co } \mathcal{B}} \subset S^{-1} \mathcal{E}$.

An $(\mathcal{E}, \mathcal{B})$-Hopf module is an $\mathcal{E}$-module $M$, with $\mathcal{B}$-coaction $\rho_{M}$, so that $\rho_{M}(e m)=\rho(e) \rho_{M}(m)$ for all $e \in \mathcal{E}$ and $m \in M$. In commutative case, Hopf modules correspond to $B$-equivariant quasicoherent sheaves over $\operatorname{Spec} \mathcal{E}$. They form a category commonly denoted by $\mathcal{E}^{\mathcal{B}}$.

A flat localization functor $Q$ on $\mathcal{E}$ - Mod is $\rho$-compatible if there is a (unique) functor $Q^{\mathcal{B}}$ on the category $\mathcal{E}^{\mathcal{B}}$ agreeing with $Q$ after forgetting the comodule structures.

DEFINITION 1. ([37, 40]) $A$ Zariski locally trivial principal $\mathcal{B}-$ bundle is an $\mathcal{E}$-comodule algebra $(\mathcal{E}, \rho)$ for which there exists a Zariski local trivialization. A Zariski local trivialization of $(\mathcal{E}, \rho)$ consists of

- a finite cover $\left\{\left(\iota_{\lambda}, S_{\lambda}^{-1} \mathcal{E}\right)\right\}_{\lambda \in \Lambda}$ of $\mathcal{E}$ by $\rho$-compatible Ore localizations, and

- a family $\left\{\gamma_{\lambda}: \mathcal{B} \rightarrow S_{\lambda}^{-1} \mathcal{E}\right\}_{\lambda \in \Lambda}$ of $\mathcal{B}$-comodule algebra maps.

Here the $\mathcal{B}$-comodule structure on $\mathcal{E}_{\lambda}$ is the one induced by $\rho$ compatibility. Maps $\gamma_{\lambda}$ are, in commutative case, induced by trivializing sections (cf. (2) in [37]), and we view $\gamma_{\lambda}$ as an algebraic replacement for trivializing sections. We discuss some generalizations in [40].

We now sketch how these fibre bundles may be understood as indeed being objects over a quantum quotient space $\left(X, \mathcal{O}_{X}\right)$.

An additive functor $f^{*}: \mathcal{A} \rightarrow \mathcal{B}$ between Abelian categories is ([32])

- continuous if it has a right adjoint, say $f_{*}$;

- flat if it is continuous and exact;

- almost affine if it is continuous and its right adjoint $f_{*}$ is faithful and exact;

- affine if it is almost affine, and its right adjoint $f_{*}$ has its own right adjoint, say $f$ !

Consider the category $\underline{C A C a t}_{*}$ whose objects are pairs of the form $\left(\mathcal{A}, \mathcal{O}_{\mathcal{A}}\right)$ where $\mathcal{A}$ is a (small in a fixed universe) Abelian category, and $\mathcal{O}_{\mathcal{A}}$ is an object in $\mathcal{A}$, and where $\operatorname{Hom}(\mathcal{A}, \mathcal{B})$ is the set of all (additive) continuous functors $f^{*}$ from $\mathcal{B}$ to $\mathcal{A}$, equipped with a distinguished isomorphism $\phi$ sending $f^{*}\left(\mathcal{O}_{\mathcal{B}}\right)$ to $\mathcal{O}_{\mathcal{A}}$. A morphism in $\underline{\text { CACat }}_{*}$ is flat, (almost) affine etc. if its underlying "inverse image" functor is. A cover is (almost) affine etc. if each morphism in the cover is such. 
A (relative) quasischeme $\mathcal{A}$ over $\mathcal{C}$ is a morphism $g: \mathcal{A} \rightarrow \mathcal{C}$ in $\underline{C A C a t}_{*}$, for which there is an almost affine cover $\left\{Q_{\mu}^{*}\right\}_{\mu \in M}$ by flat localizations where all $g_{*} \circ Q_{\mu *}$ are exact and faithful. It is a (relative noncommutative) scheme ([32]) if the cover $\left\{Q_{\mu}^{*}\right\}_{\mu \in M}$ in the definition can be actually chosen affine, with all $Q_{\mu}^{*} \circ g^{*}$ affine as well.

If $\mathcal{E}$ has a trivializing cover $\left\{\left(\iota_{\lambda}, S_{\lambda}^{-1} \mathcal{E}, \gamma_{\lambda}\right)\right\}_{\lambda \in \Lambda}$, which is in the same time the affine cover in the definition of a relative scheme over the (category of modules over) ground ring $\mathbf{k}$, then the category $\mathcal{E}^{\mathcal{B}}$ has the structure of noncommutative scheme $\left(X, \mathcal{O}_{X}\right)$ over $\mathbf{k}$ as well. Namely, the gluing of Hopf modules over charts of the cover reduces to the globalization of ordinary modules (analogue of the statement that if we glue equivariant sheaves over charts on a manifold to a global sheaf then this sheaf is automatically equivariant). It follows that the localizations $Q_{\lambda}^{\mathcal{B}}$ (which exist and are determined by compatibility and $Q_{\lambda}$ ) form an affine cover of $\mathcal{E} \mathcal{M}^{\mathcal{B}}$, and the local triviality ensures (cf. 35], and [37, Section 9) that for each $\lambda$ there is a natural SchneIDER's equivalence of categories between the localized category $\mathcal{E}_{\mathcal{\lambda}}^{\mathcal{B}}$ and the category $\left(\mathcal{E}_{\lambda}\right)^{\mathrm{co} \mathcal{B}}-$ Mod of modules over the algebra of localized coinvariants. By descent, its structure sheaf $\mathcal{O}_{X}$ corresponds to the family $\left\{\mathcal{U}^{\lambda}\right\}_{\lambda \in \Lambda}$ of algebras of localized coinvariants $\mathcal{U}^{\lambda}=\left(\mathcal{E}_{\lambda}\right)^{\mathrm{co} \mathcal{B}}$. If $\mathcal{U}:=\mathcal{E}^{\mathrm{coB}}$ is the algebra of global coinvariants ("affine quotient"), then $\left(X, \mathcal{O}_{X}\right)$ is actually a relative scheme over $\mathcal{u} \mathcal{M}$, hence a fortiori over $\mathbf{k}$.

\section{Quantum associated bundles}

For any k-coalgebra $C$ (e.g. $C=\mathcal{B})$, denote by $\mathcal{M}^{C}\left(\right.$ resp. $\left.{ }^{C} \mathcal{M}\right)$ the category of right (left) $C$-comodules. Cotensor product is a bifunctor $\square=\square^{C}: \mathcal{M}^{C} \times{ }^{C} \mathcal{M} \rightarrow{ }_{\mathrm{k}} \mathcal{M}$ which is given on objects as $N \square M:=$ $\operatorname{ker}\left(\rho_{N} \otimes \operatorname{id}_{M}-\operatorname{id}_{N} \otimes \rho_{M}\right)$. The same formula defines the bifunctor $\square: \mathcal{E}_{\mathcal{M}} \mathcal{M}^{\mathcal{B}} \times{ }^{\mathcal{B}} \mathcal{M} \rightarrow \mathcal{E} \mathcal{M}$. If $D$ is flat as a $\mathbf{k}$-module (e.g. $\mathbf{k}$ is a field), and $N$ a left $D$ - right $C$-bicomodule, then the cotensor product $N \square M$ is a $D$-subcomodule of $N \otimes_{\mathbf{k}} M$. In particular, if $\pi: D \rightarrow C$ is a surjection of coalgebras then $D$ is a left $D$ - right $C$-bicomodule via $\Delta_{D}$ and $(\mathrm{id} \otimes \pi) \circ \Delta_{D}$ respectively, hence $\operatorname{Ind}_{C}^{D}:=D \square^{C}$ is a functor from left $C$ - to left $D$-comodules called the induction from $C$ to $D$.

Consider for a moment functor $\mathcal{E} \otimes_{\mathrm{k}-}:{ }^{\mathcal{B}} \mathcal{M} \rightarrow{ }_{\mathbf{k}} \mathcal{M}$ (the superscript is intended!). Given a map $\gamma: \mathcal{B} \rightarrow \mathcal{E}$ of $\mathcal{B}$-comodules for which there is a convolution-inverse $\gamma^{-1}$, define natural transformations of functors $\kappa^{\gamma}, \bar{\kappa}^{\gamma}: \mathcal{E} \otimes_{\mathbf{k}-} \rightarrow \mathcal{E} \otimes_{\mathbf{k}-}$ by $\kappa_{M}^{\gamma}\left(\sum_{i} e_{i} \otimes m_{i}\right)=\sum_{i} e_{i} \gamma\left(m_{i(-1)}\right) \otimes m_{(0)}$ and $\bar{\kappa}^{\gamma}=\kappa_{\gamma^{-1}}$. If $\gamma$ is a map of $\mathcal{B}$-comodule algebras, then $\gamma^{-1}=\gamma \circ S$. In that case, restrict the natural transformation $\kappa^{\gamma}$ to the subfunctor $\mathcal{E}^{\mathrm{coB}} \otimes_{\mathbf{k}}$ - and $\bar{\kappa}^{\gamma}$ to the subfunctor $\mathcal{E} \square^{\mathcal{B}}$ - and denote the restrictions 
simply by $\kappa^{\gamma} \mid$ and $\bar{\kappa}^{\gamma} \mid$. For any natural transformation of functors with values $\Phi: F \rightarrow G$ in a (say) Abelian category $G$, denote by $\operatorname{Im} \Phi: F \rightarrow$ $G$ the functor $M \mapsto \operatorname{Im} \Phi_{M}(F(M))$.

LEMMA 1. (a) $\kappa^{\gamma} \circ \bar{\kappa}^{\gamma}=\operatorname{Id}_{\mathcal{E} \otimes_{\mathbf{k}-}}=\bar{\kappa}^{\gamma} \circ \kappa^{\gamma}$;

(b) $\operatorname{Im}\left(\kappa^{\gamma} \mid\right)=\mathcal{E} \square^{\mathcal{B}}$ - and $\operatorname{Im}\left(\bar{\kappa}^{\gamma} \mid\right)=\mathcal{E}^{\operatorname{co} \mathcal{B}} \otimes_{\mathbf{k}-}$.

Proof. (a) follows by calculation: e.g. the right-hand equality by

$$
\begin{aligned}
\bar{\kappa}^{\gamma}\left(\kappa^{\gamma}\left(\sum_{i} f_{i} \otimes v_{i}\right)\right) & =\bar{\kappa}^{\gamma}\left(\sum_{i} f_{i} \gamma\left(v_{i(-1)}\right) \otimes v_{i(0)}\right) \\
& =\sum_{i} f_{i} \gamma\left(v_{i(-2)}\right) \gamma^{-1}\left(v_{i(-1)}\right) \otimes v_{i(0)} \\
& =\sum_{i} f_{i} \epsilon\left(v_{i(-1)}\right) \otimes v_{i(0)}=\sum_{i} f_{i} \otimes v_{i} .
\end{aligned}
$$

(a) implies that the two equalities in part (b) are equivalent. By the definition, $\operatorname{Im}\left(\kappa_{M}^{\gamma} \mid\right)$ consists of the elements of the form $\sum_{j} f_{j} \gamma\left(m_{j(-1)}\right) \otimes$ $m_{j(0)}$ where $F=f_{j} \in \mathcal{E}^{\mathrm{co} \mathcal{B}}$ and $m_{j} \in M$. The coaction axiom, $\rho\left(f_{j}\right)=$ $f_{j} \otimes 1$, and the requirement that $\gamma$ is a map of comodules imply

$$
\left(\operatorname{id}_{\mathcal{E}} \otimes \rho_{M}\right)(F)=\sum_{j} f_{j} \gamma\left(m_{j(-2)}\right) \otimes m_{j(-1)} \otimes m_{j(0)}=\left(\rho_{\mathcal{E}} \otimes \operatorname{id}_{M}\right)(F)
$$

hence $F \in \mathcal{E} \square^{\mathcal{B}} M$ and $\operatorname{Im}\left(\kappa_{M}^{\gamma} \mid\right) \subset \mathcal{E} \square^{\mathcal{B}} M$.

To prove $\operatorname{Im}\left(\kappa_{M}^{\gamma} \mid\right) \supset \mathcal{E} \square^{\mathcal{B}} M$ assume the contrary $-\exists H \in \mathcal{E} \square^{\mathcal{B}} M \subset$ $\mathcal{E} \otimes_{\mathbf{k}} M$ not in the image. Recall that existence of $\gamma$ implies (e.g. 27, Ch. 4; 25]; or 37], Sec. 6) that id $\otimes \gamma$ followed by multiplication map in $\mathcal{E}$ is an isomorphism $\mathcal{E}^{\mathrm{co} \mathcal{B}} \otimes \mathcal{B} \cong \mathcal{E}$ with inverse which we denote here $\phi^{\gamma}$. In particular, elements of $\mathcal{E}$ are additive combinations of elements of the form $f \gamma(\zeta)$ where $f \in \mathcal{E}^{\mathrm{co} \mathcal{B}}$ and $\zeta \in \mathcal{B}$. Hence $\exists n \in \mathbb{Z}$, $\exists f_{i} \in \mathcal{E}^{\mathrm{co} \mathcal{B}}, \zeta_{i} \in \mathcal{B}, i=1, \ldots, n$ such that $H=\sum_{i} f_{i} \gamma\left(\zeta_{i}\right) \otimes m_{i}$.

Since the coaction $\rho_{\mathcal{E}}$ is an algebra map and $\gamma$ an intertwiner, then $\rho\left(f_{i} \gamma\left(\zeta_{i}\right)\right)=f_{i} \gamma\left(\zeta_{i(1)}\right) \otimes \gamma\left(\zeta_{i(2)}\right)$. Since $H \in \mathcal{E} \square^{\mathcal{B}} M$ we have

$$
\phi^{\gamma}\left(\rho_{\mathcal{E}} \otimes \mathrm{id}\right)(H)=\phi^{\gamma}\left(\mathrm{id} \otimes \rho_{M}\right)(H) .
$$

Evaluated and in Sweedler notation equation 1 reads

$$
\sum_{i} f_{i} \otimes \zeta_{i} \otimes m_{i(-1)} \otimes m_{i(0)}=\sum_{i} f_{i} \otimes \zeta_{i(1)} \otimes \zeta_{i(2)} \otimes m_{i} .
$$

Apply id $\otimes \epsilon \otimes$ id $\otimes$ id to this equation we obtain

$$
\begin{gathered}
\sum_{i} f_{i} \epsilon\left(\zeta_{i}\right) \otimes m_{i(-1)} \otimes m_{i(0)}=\sum_{i} f_{i} \otimes \zeta_{i} \otimes m . \\
\operatorname{Im} \kappa_{M}^{\gamma} \mid \ni \sum_{i} f_{i} \epsilon\left(\zeta_{i}\right) \gamma\left(m_{i(-1)}\right) \otimes m_{i(0)}=\sum_{i} f_{i} \gamma\left(\zeta_{i}\right) \otimes m_{i}=H . \quad \Rightarrow \Leftarrow
\end{gathered}
$$


By abuse of notation, let $\kappa^{\gamma} \mid: \mathcal{E}^{\mathrm{co} \mathcal{B}} \otimes_{\mathbf{k}-} \rightarrow \mathcal{E} \square_{-}$denote also the corestriction of $\kappa^{\gamma} \mid$ onto the image functor $\mathcal{E} \square_{-}$, and alike for $\bar{\kappa}^{\gamma}$. The lemma easily implies that $\kappa^{\gamma} \mid$ is an equivalence of subfunctors with inverse $\bar{\kappa}^{\gamma} \mid$. That is, the pair of natural transformations $\left(\kappa^{\gamma}\left|, \bar{\kappa}^{\gamma}\right|\right)$ extends to a pair of mutually inverse natural autoequivalences of $\mathcal{E} \otimes_{\mathbf{k}_{-}}$, namely $\left(\kappa^{\gamma}, \bar{\kappa}^{\gamma}\right)$.

We apply this discussion to our localization picture. For any local trivialization $\Lambda=\left\{\iota_{\lambda}, \mathcal{E}_{\lambda}, \gamma_{\lambda}\right\}_{\lambda \in \Lambda}$ of $\mathcal{E}$, we have the natural transformations $\kappa_{\lambda}=\kappa^{\gamma_{\lambda}}, \bar{\kappa}_{\lambda}=\kappa^{\gamma_{\lambda} \circ S}$ for all $\lambda \in \Lambda$, and the coproducts $\mathcal{E}_{\lambda} \square M$ are locally "identified" to $\mathcal{E}_{\lambda}^{\text {co } \mathcal{B}} \otimes_{\mathbf{k}} M$. Introduce functor $\Gamma_{\lambda} \xi_{-}$: $\mathcal{B}^{\mathcal{M}} \rightarrow{ }_{\mathbf{k}} \mathcal{M}$ by $M \mapsto \Gamma_{\lambda} \xi_{M}=\mathcal{E}_{\lambda}^{\text {co } \mathcal{B}} \otimes_{\mathbf{k}} M$ and similarily, for consecutive localizations, $\Gamma_{\lambda \mu \ldots} \xi_{-}$. Define the natural transformations $\kappa_{\lambda \lambda^{\prime}}^{\lambda}$ by the compositions

$$
\kappa_{\lambda \lambda^{\prime} M}^{\lambda}: \Gamma_{\lambda} \xi_{M} \stackrel{\kappa_{\lambda, M}}{\longrightarrow} \mathcal{E}_{\lambda} \square M \stackrel{\iota_{\lambda \lambda^{\prime}}^{\lambda} \square M}{\longrightarrow} \mathcal{E}_{\lambda \lambda^{\prime}} \square M .
$$

and similarily define $\kappa_{\lambda \lambda^{\prime}}^{\lambda^{\prime}}$ using $\iota_{\lambda \lambda^{\prime}}^{\lambda^{\prime}}$. Finally, natural transformations

$$
\mathcal{K}_{\Lambda \Lambda}: \prod_{\lambda \in \Lambda} \Gamma_{\lambda} \xi_{-} \rightarrow \prod_{\left(\lambda, \lambda^{\prime}\right) \in \Lambda \times \Lambda} \mathcal{E}_{\lambda \lambda^{\prime}} \square
$$

are defined by $\operatorname{pr}_{\lambda \lambda^{\prime}} \circ \mathcal{K}_{\Lambda \Lambda}=\prod_{\lambda \in \Lambda} \kappa_{\lambda \lambda^{\prime}}^{\lambda} \circ \operatorname{pr}_{\lambda}$ where $\operatorname{pr}_{\mu}: \prod_{\lambda} \Gamma_{\lambda} \xi_{-} \rightarrow \Gamma_{\mu} \xi_{-}$ are the natural projection transformations and $\operatorname{pr}_{\lambda \lambda^{\prime}}$ alike; and similarily define $\mathcal{K}_{\Lambda \Lambda}^{\prime}$ by using $\kappa_{\lambda \lambda^{\prime}}^{\lambda^{\prime}}$. The global sections of associated vector bundle functor $\Gamma_{\Lambda} \xi_{-}$is the subfunctor of $\prod_{\lambda \in \Lambda} \Gamma_{\lambda} \xi_{-}$such that the fork diagram

$$
\Gamma_{\Lambda} \xi_{-} \stackrel{\text { in }}{\longrightarrow} \prod_{\lambda \in \Lambda} \Gamma_{\lambda} \xi_{-} \stackrel{\stackrel{\mathcal{K}_{\Lambda \Lambda}}{\longrightarrow}}{\underset{\mathcal{K}^{\prime} \Lambda \Lambda}{\longrightarrow}} \prod_{\left(\lambda, \lambda^{\prime}\right) \in \Lambda \times \Lambda} \mathcal{E}_{\lambda \lambda^{\prime}} \square_{-}
$$

is an equalizer diagram of natural transformations.

THEOREM 1. Functors $\Gamma_{\Lambda} \xi_{-}$are naturally equivalent for different local trivializations $\Lambda$. There is a natural equivalence $\mathcal{K}_{\Lambda}: \Gamma_{\Lambda} \xi_{-} \rightarrow \mathcal{E} \square$ making the following diagram sequentially commute:

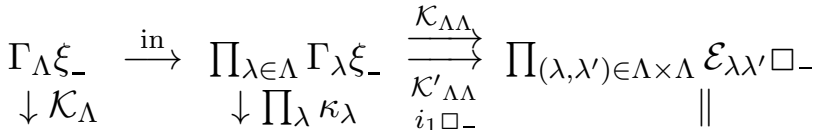

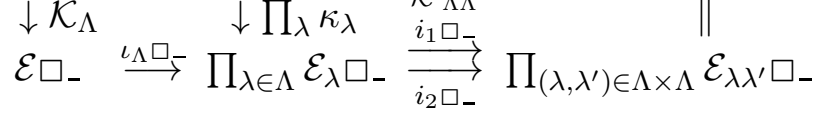

Proof. The square on the right is manifestly commutative by the construction of the maps involved. Since upper and lower fork diagrams are equalizer diagrams, and the right vertical arrows natural equivalences, 
the transformation $\mathcal{K}_{\Lambda}$ exists and is uniquely defined by the rest of the diagram.

As a consequence, given two different local trivializations $\Lambda, \Lambda^{\prime}$ the transformation $\mathcal{K}_{\Lambda^{\prime}} \circ \mathcal{K}_{\Lambda}: \Gamma_{\Lambda} \xi_{-} \stackrel{\cong}{\longrightarrow} \Gamma_{\Lambda^{\prime}} \xi_{-}$is a canonical isomorphism of functors. Hence for fixed $M$, we can denote by $\Gamma \xi_{M}$ the equivalence class of pairs of the form $\left(\Lambda, \Gamma_{\Lambda} \xi_{M}\right)$.

Actually, for more general localizations, that is for $Q_{\mu}^{*}$, corresponding to any $\rho$-compatible radical filter $\mathcal{F}_{\mu}$, and given Zariski local trivialization $\Lambda$, we can define $\Gamma_{\mu \Lambda} \xi_{-}$by essentially the same procedure for $\mathcal{E}_{\mu}$ instead of $\mathcal{E}$, and again, it is independent of the choice of $\Lambda$. The lattice $\mathcal{L}_{\mathcal{E}}^{\mathcal{B}}$ of $\rho$-compatible radical filters is identified with the lattice $\mathbf{L}_{X}$ of flat localizations of the quotient noncommutative scheme $X$. Thus we obtain a bifunctor $\Gamma_{-} \xi_{-}$, i.e. a presheaf $\mu \mapsto \Gamma_{\mu} \xi_{-}$of functors $\Gamma_{\mu} \xi_{-}: M \mapsto \Gamma_{\mu} \xi_{M}$ over $\mathbf{L}_{X}$. One can consider more general situations where the global space is not described by only one algebra, but the Zariski principal bundles make sense, and the presheaf $\mu \mapsto \Gamma_{\mu} \xi_{-}$of functors over $\mathbf{L}_{X}$ still makes sense, whereas the global cotensor product does not. Alternatively, we may construct the associated bundles by means of transition matrices (40]).

Quantum line bundles. Let us specialize now to $\mathbf{k}=\mathbb{C}$, choose a group-like element $\chi \in \mathcal{B}$ and consider the 1-dimensional left comodule $M=\mathbb{C}_{\chi}$ given by $\rho_{M}(m)=m \otimes \chi$. Denote the "line bundle" $\xi_{M}$ by $L_{\chi}$. Its space of sections $\Gamma L_{\chi}$ can be identified with a $\mathbb{C}$-subspace of $\prod_{\lambda} \mathcal{E}_{\lambda}$. Namely, write explicitly maps $\mathcal{K}_{\Lambda \Lambda^{\prime}}$ and $\mathcal{K}^{\prime}{ }_{\Lambda \Lambda^{\prime}}$, and use the identifications $\mathcal{E}_{\lambda} \otimes M \cong \mathcal{E}_{\lambda}$ in expressing (2) to obtain

$$
\Gamma L_{\chi} \cong\left\{\begin{array}{l|c}
f=\prod_{\lambda \in \Lambda} f_{\lambda} \mid \begin{array}{c}
f_{\lambda} \gamma_{\lambda}(\chi)=f_{\lambda^{\prime}} \gamma_{\lambda^{\prime}}(\chi) \\
\forall \lambda, \lambda^{\prime} \text { in } \mathcal{E}_{\lambda \lambda^{\prime}} \text { and in } \mathcal{E}_{\lambda^{\prime} \lambda}
\end{array}
\end{array}\right\}
$$

THEOREM 2. $\Gamma L_{\chi}$ is naturally isomorphic to the cotensor product $\mathcal{E} \square_{\mathcal{B}} M$ as a $\mathbf{k}$-vector space.

Now assume $\mathcal{E}$ is an algebra in category of left $D$ - right $\mathcal{B}$-comodules where $D$ is a $\mathbf{k}$-coalgebra, flat as $\mathbf{k}$-module. Particular case of importance to us is when $\mathcal{G}=\mathcal{E}=D$ is a Hopf k-algebra and $\pi: \mathcal{G} \rightarrow \mathcal{B}$ is a surjective homomorphism of Hopf algebras, with natural right $\mathcal{B}$-comodule structure $($ id $\otimes \pi) \circ \Delta_{\mathcal{G}}$.

Now repeat the arguments preceding Theorem 1, but now with the (compatible with other data) left $D$-comodule structure added. More specifically, we consider functor $\mathcal{E} \otimes_{\mathbf{k}}-:{ }^{\mathcal{B}} \mathcal{M} \rightarrow{ }^{D} \mathcal{M}$, and the corresponding versions of natural transformations $\kappa^{\gamma}$ etc. and conclude that the 'new' $\kappa^{\gamma}$ is a natural autoequivalence of the functor 
$\mathcal{E} \otimes_{\mathbf{k}-}:{ }_{\mathcal{E}}^{\mathcal{B}} \mathcal{M} \rightarrow{ }^{D} \mathcal{M}$ with inverse $\bar{\kappa}^{\gamma}$, inducing the equivalence of subfunctors between $\mathcal{E}^{\mathcal{B}}$ - and $\mathcal{E}^{\mathrm{co} \mathcal{B}} \otimes_{\mathbf{k}}$ - Furthermore, construct the left $D$-comodule enrichment of sections modules $\Gamma_{\lambda} \xi_{-}, \Gamma_{\Lambda} \xi_{-}$and notice that the morphisms $\mathcal{K}_{\Lambda \Lambda}$ etc. in the corresponding commutative diagrams respect the left $D$-comodule structure. Hence we have

THEOREM 3. If $C$ is a coalgebra and $\mathcal{E}$ is left $D$-right $G$-bicomodule, then the equivalences in Theorems 1, 2 respect the D-comodule structure. In particular, if $\mathcal{G}=D=\mathcal{E}$ is a Hopf algebra, and $\mathcal{B}$ a quantum subgroup, then $\Gamma L_{\chi}$ is isomorphic to the induced $\mathcal{G}$-comodule from $\mathcal{B}$.

\section{Background: Perelomov coherent states}

Perelomov coherent states generalize the Schrödinger coherent states to the Lie group setting $(29,28])$.

We use the geometric language of [30; cf. also [6].

Let $G^{\mathbb{C}}$ be a complex connected semisimple Lie group with compact real form $G$, and a Borel subgroup $B$. We will often view these groups as affine algebraic groups over $\mathbb{C}$. Let $\chi: B \rightarrow \mathbb{C}$ be a character of $B$ and $\mathbb{C}_{\chi}$ the corresponding 1-dimensional $B$-module. The projection $p$ : $G^{\mathbb{C}} \rightarrow G^{\mathbb{C}} / B$ defines a principal $B$-bundle. The associated bundle $L_{\chi}=$ $G^{\mathbb{C}} \times{ }_{\chi} \mathbb{C}_{\chi}$ with the projection $p_{L}: L_{\chi} \rightarrow G^{\mathbb{C}} / B$. The left action of $G$ on $G^{\mathbb{C}}$ induces an action of $G$ on $L_{\chi}$ and the formula $\left(g_{*} s\right)(x)=g s\left(g^{-1} x\right)$ defines an action of $G^{\mathbb{C}}$ on the space $V_{\chi}=\Gamma L_{\chi}$ of holomorphic sections of $L_{\chi}$ which is by BOREL-WEIL theorem, an irreducible unitarizable $G$-module. An invariant unitary product on $\Gamma L_{\chi}$, antilinear in $1^{\text {st }}$ and linear in $2^{\text {nd }}$ argument, is denoted $\langle\mid\rangle$.

Consider a (holomorphic) section $s \in \Gamma L_{\chi}$ and a nonzero point $q$ in some fiber $p_{L}^{-1}(x)$. Then

$$
s(x)=s\left(p_{L}(q)\right)=l_{q}(s) q,
$$

for some number $l_{q}(s)$. The correspondence

$$
s \mapsto l_{q}(s), \quad l_{q}: \Gamma L_{\chi} \rightarrow \mathbb{C},
$$

is a continuous linear functional. Using Riesz's theorem, we infer the existence of an element

$$
e_{q} \in \Gamma L_{\chi} \text { such that } l_{q}(s)=\left\langle e_{q} \mid s\right\rangle .
$$

The vectors (sections) of the form $e_{q} \in \Gamma L_{\chi}$ are called coherent vectors. Corresponding projective classes are called coherent states. 
PROPOSITION 1. ([30, [36]) (i) $e_{g q}=g_{*} e_{q}$ for all $g \in G^{\mathbb{C}}$.

(ii) $e_{c q}=\bar{c}^{-1} e_{q}$ for all $c \in \mathbb{C}$.

(iii) Coherent states i.e. the projective classes of all coherent vectors belong to the same projective orbit.

(iv) The set of all $e_{q}$ where $q \in\left(p_{L}\right)^{-1}\left(1_{G} B\right)$ agrees with the set (ray) of all heighest weight vectors in $V_{\chi}$ for fixed $B$.

(v) The set of all $e_{q}$ where $q \in\left(p_{L}\right)^{-1}(u)$ for fixed $u \in G^{\mathbb{C}} / B$ is the heighest weight space for some subgroup of $G^{\mathbb{C}}$ conjugated to $B$.

COROLLARY 1. Let $U \subset G^{\mathbb{C}} / B$ be an open set, $q \in L_{\chi}$ given, and $t: U \rightarrow G^{\mathbb{C}}$ a section of the principal B-bundle $p^{-1}(U) \rightarrow U$. For each $g \in p^{-1}(U)$ there is a unique decomposition $g=t(g B) b$ such that $b \in B$. The following "homogeneity" formula holds:

$$
g e_{q}=\chi^{-1}(b) e_{t(g B) q}
$$

Proof. We have $g_{*} e_{q}=t(g B)_{*} b_{*} e_{q}=t(g B)_{*} \chi^{-1}(b) e_{q}$ by (6); taking into account that $\chi^{-1}(b)$ is a scalar, this equals to $\chi^{-1}(b) t(g B)_{*} e_{q}$, hence by (i) of the Proposition [1] also to $\chi^{-1}(b) e_{t(g B) q}$.

DEFINITION 2. The local family of coherent vectors corresponding to the triple $(U, t, q)$ is the map

$$
C_{(U, t, q)}: U \rightarrow V_{\chi} \equiv \Gamma L_{\chi}, \quad C_{(U, t, q)}:[g] \mapsto e_{t([g]) q} .
$$

For any $w$ in the Weyl group $W$ of $G$, there is a Zariski open subset $G_{w}^{\mathbb{C}} \subset G^{\mathbb{C}}$ consisting of all $g \in G^{\mathbb{C}}$ for which there exists (automatically unique) $w$-Gauss decomposition $g=w y b$ where $y \in G^{\mathbb{C}}$ belongs to the unipotent subgroup of the opposite Borel $B^{\prime}$, and $b \in B$. Set $G_{w}^{\mathbb{C}}$ is also $B$-invariant, hence a total space of the restricted fibration over a Zariski open subset $G_{w}^{\mathbb{C}} / B \subset G^{\mathbb{C}} / B$. Define the local section $t_{w}$ : $G_{w}^{\mathbb{C}} / B \rightarrow G_{w}^{\mathbb{C}} \subset G^{\mathbb{C}}$ by $t_{w}([g])=w y$ where $g=w y b$ as above. We denote

$$
C_{w}:=C_{\left(w, v_{0}\right)}:=C_{\left(G_{w}^{\mathrm{C}} / B, t_{w}, q\right)}
$$

where $v_{0}=e_{q}$ is a fixed highest weight vector in $V_{\chi}$. The collection of maps $\left\{C_{w}, w \in W\right\}$ will be generalized to the quantum group setting below. They can be viewed as $C_{w} \in \mathcal{O}\left(G_{w}^{\mathbb{C}} / B\right) \otimes V_{\chi}$ where $\mathcal{O}\left(G_{w}^{\mathbb{C}} / B\right)$ is the complex algebra of all algebraic functions on $G_{w}^{\mathbb{C}} / B$.

In this particular case, Corollary 1 becomes

PROPOSITION 2. If $g=w y b$ is the Gauss decomposition in $G_{w}$ then for all $g \in G$

$$
g v_{0}=\chi^{-1}(b) C_{w}(g B),
$$

and $C_{w}$ is the unique element in $\mathcal{O}\left(G_{w}^{\mathbb{C}} / B\right) \otimes V_{\chi}$ for which this holds. 


\section{Quantum coherent states and localizations}

DEFINITION 3. Let $\chi$ be a group-like element in a Hopf algebra $\mathcal{B}$, and $(V, \rho)$ a right $\mathcal{B}$-comodule. A $\chi$-coinvariant in $V$ is an element $v_{\chi} \in V$ such that $\rho v_{\chi}=v_{\chi} \otimes \chi$.

Let $\pi: \mathcal{G} \rightarrow \mathcal{B}$ be a surjective homomorphism of Hopf algebras. We say that $(\pi, \mathcal{B})$ is a quantum subgroup of $\mathcal{G}$. Every $\mathcal{G}$-comodule (resp. comodule algebra) $(V, \rho)$ is a $\mathcal{B}$-comodule (comodule algebra) via $\rho_{\mathcal{B}}=($ id $\otimes \pi) \circ \rho$. In particular, $\mathcal{B}$ coacts on $\mathcal{G}$ by $($ id $\otimes \pi) \circ \Delta_{\mathcal{G}}$ and this coaction makes $\mathcal{G}$ a left $\mathcal{B}$-comodule algebra and similarily for the right coactions. In particular, $\mathcal{G}$ can be viewed as left-right $\mathcal{B}$ $\mathcal{G}$-bicomodule ${ }^{\mathcal{B}} \mathcal{G}^{\mathcal{G}}$. Hence to each $\mathcal{B}$-comodule $V$ one can attach an induced $\mathcal{G}$-comodule by the formula $\operatorname{Ind}_{\mathcal{B}}^{\mathcal{G}} V=V \square^{\mathcal{B}} \mathcal{G}$. This defines the induction functor $\operatorname{Ind}_{\mathcal{B}}^{\mathcal{G}}$ which is left adjoint to the restriction functor $(V, \rho) \mapsto\left(V, \rho_{\mathcal{B}}\right)$ (Frobenius reciprocity for comodules).

DEFINITION 4. Let $(V, \rho)$ be any $\mathcal{G}$-comodule and $(\pi, \mathcal{B})$ a quantum subgroup of $\mathcal{G}$. $A$ weight covector of weight $\chi$, is any $\chi$-coinvariant for $\mathcal{B}$-coaction $\rho_{\mathcal{B}}$ in $V$ i.e.

$$
(\mathrm{id} \otimes \pi) \rho v_{\chi}=v_{\chi} \otimes \chi .
$$

Let $\left(V_{\chi}, \rho\right)=\operatorname{Ind}_{\mathcal{B}}^{\mathcal{G}} \mathbb{C}_{\chi}$ be the induced right $\mathcal{G}$-comodule induced from the 1-dimensional comodule $z \mapsto z \otimes \chi$.

DEFINITION 5. A *-involution on a $\mathbb{C}$-bialgebra $H$ is an antilinear map * $: H \rightarrow H$, for which $(a b)^{*}=b^{*} a^{*}, \Delta\left(a^{*}\right)=\sum a_{(1)}^{*} \otimes a_{(1)}^{*}$ and $\epsilon\left(a^{*}\right)=\overline{\epsilon(a)}$. A pair $(H, *)$ is called a real form of $H$.

LEMMA 2. (Schur's lemma for comodules) Let $C$ be a coalgebra over $\mathbb{C}$ and $(V, \rho)$ a right $C$-comodule. If $(V, \rho)$ is finite-dimensional and simple (no coinvariant subspaces), then every $C$-comodule map $A: V \rightarrow V$ equals $\alpha \cdot \mathrm{id}_{V}$ for some $\alpha=\alpha_{A} \in \mathbb{C}$.

DEFINITION 6. ([2]) Let $H$ be a Hopf *-algebra. An inner product $\langle\cdot \mid \cdot\rangle$ on a right $H$-comodule $V$ is a coinvariant inner product iff

$$
\langle w \mid z\rangle 1_{H}=\sum\left\langle w_{(0)} \mid z_{(0)}\right\rangle z_{(1)} w_{(1)}^{*}
$$

An $H$-comodule which is a Hilbert space via a coinvariant inner product will be called a right unitary $H$-comodule.

Consider a real form of a Hopf algebra $\mathcal{G}$ with the following data: 
- (D1) A surjective map of Hopf algebras $\pi: \mathcal{G} \rightarrow \mathcal{B}$.

- (D2) A group-like element $\chi \in \mathcal{B}$.

- (D3) A coinvariant inner product on $V_{\chi}$.

- (D4) A weight covector $v_{\chi} \in V_{\chi}$ with norm 1.

- (D5) A Zariski local trivialization $\Lambda=\left\{\lambda=\left(\iota_{\lambda}, S_{\lambda}^{-1} \mathcal{G}, \gamma_{\lambda}\right)\right\}_{\lambda \in \Lambda}$ of $\mathcal{G}$ as a right $\mathcal{B}$-comodule algebra.

From now on, $V$ will be a comodule over the real form of $\mathcal{G}$ with fixed unitary equivalence $V \cong V_{\chi}$ which we often treat as an identification. Denote by $V^{\text {triv }}$ the trivial $\mathcal{G}$-comodule with the same underlying vector space as $V$.

DEFINITION 7. Let (D1-5) be given and $\lambda \in \Lambda$. A (Zariski-) local family of coherent vectors in $\lambda$ or a polynomial coherent vector $^{1}$ in $\lambda$ is an element $C_{\lambda} \in V \otimes \mathcal{G}_{\lambda}^{\text {co } \mathcal{B}}$ such that

$$
\rho_{\lambda} v_{\chi}=C_{\lambda} \gamma_{\lambda}(\chi)
$$

holds in $V \otimes \mathcal{G}_{\lambda}$ where $\gamma_{\lambda}(\chi)$ on the right multiplies the second tensor factor in $C_{\lambda}$ and $\rho_{\lambda}$ is the localized $\mathcal{B}$-coaction (id $\left.\otimes \iota_{\lambda}\right) \rho$. A global family of coherent vectors is an element $C$ of $\Gamma\left(V^{\text {triv }} \otimes L_{\chi}\right)$ such that $\mathcal{K}(C)=\mathcal{K}_{\Lambda}\left(C_{\Lambda}\right)=\rho v_{\chi}$ (for one, hence any, choice of $\Lambda$ ). Then $\kappa_{\lambda}(C)=\rho_{\lambda} v_{\chi}$.

Remark. Equality (77) is a generalization of the identity (6) and related to Proposition 5.11 in [17.

PROPOSITION 3. The following are equivalent:

a) There exists a global family of coherent states $C$;

b) There exists a local trivialization $\Lambda$ of $\mathcal{E}$ such that a local family of coherent states $C_{\lambda}$ exists for each $\lambda$ in $\Lambda$;

c) For each local trivialization $\Lambda$ of $\mathcal{E}$ and each $\lambda$ in $\Lambda$ there exists a local family $C_{\lambda}$ of coherent states in $\lambda$.

Since $\mathcal{K}_{\Lambda}$ is a natural equivalence, if (a-c) are true, then the global family is unique. The same for the local family in any given local trivialization.

Proof. An exercise to the reader: use the globalization lemma and the explicit description of $\mathcal{K}_{\Lambda}$. Notice though that given only one $C_{\lambda}$ does not always suffice. Indeed, $C_{\lambda} \gamma_{\lambda}(\chi) \gamma_{\lambda^{\prime}}(S \chi)$ is a candidate for $C_{\lambda^{\prime}}$, but it does not need to extend to an element in $V \otimes \mathcal{G}_{\lambda^{\prime}}$ in general.

\footnotetext{
1 Terminological remark. 'Polynomial' because it is a polynomial in the generators of algebra $\mathcal{E}^{\text {co } \mathcal{B}}$ of localized coinvariants decorated (tensored) with coefficients in Hilbert space. This terminology is occasionally used in physics literature (in the group case, as well as in the quantum group examples, e.g. 13], p. 1382).
} 
Let us extend the product $\langle\mid\rangle$ on $V \cong V \otimes \mathbb{C} \subset V \otimes \mathcal{G}$ to a sesquilinear form

$$
\langle\mid\rangle:(V \otimes \mathcal{G}) \otimes V \rightarrow \mathcal{G}, \quad\left\langle\sum_{o} w_{i} \otimes g_{i} \mid v\right\rangle:=\sum_{i}\left\langle w_{i} \mid v\right\rangle g_{i},
$$

and analogously define $\langle\mid\rangle_{\lambda \mu \ldots}$ (often skipping the subscripts) on $V \otimes$ $\mathcal{G}_{\lambda \mu \ldots}$. In particular, for any $v \in V_{\chi}$ the expression $\left\langle C_{\lambda} \mid v\right\rangle_{\lambda}$ is an element in $\mathcal{G}_{\lambda}$. Let $\left|C_{\lambda}\right\rangle:=C_{\lambda}$ in such context.

PROPOSITION 4. For each $v \in V, \prod_{\lambda}\left\langle C_{\lambda} \mid v\right\rangle_{\lambda}$ is an element in $\Gamma_{\Lambda} L_{\chi}$, and hence, by Theorem [2, it determines an element in $V_{\chi} \cong V$.

Proof. By the definition, $\left\langle C_{\lambda} \mid v\right\rangle \in \mathcal{G}_{\lambda}^{\mathrm{co} \mathcal{B}}$. Hence, by (3), for each pair $\left(\lambda, \lambda^{\prime}\right)$, we have to check that $\left\langle C_{\lambda} \mid v\right\rangle_{\lambda} \gamma_{\lambda}(\chi)=\left\langle C_{\lambda^{\prime}} \mid v\right\rangle_{\lambda^{\prime}} \gamma_{\lambda^{\prime}}(\chi)$ in both consecutive localizations. To that aim observe that

$$
\left\langle\rho_{\lambda} v_{\chi} \mid v\right\rangle_{\lambda}=\left\langle C_{\lambda} \cdot\left(1 \otimes \gamma_{\lambda} \chi\right) \mid v\right\rangle_{\lambda}=\left\langle C_{\lambda} \mid v\right\rangle_{\lambda} \gamma_{\lambda}(\chi) .
$$

Then observe, that symbol $\langle\mid v\rangle=\langle, v\rangle \otimes$ id commutes with the localizations, in the sense that (id $\left.\otimes \iota_{\lambda, \lambda^{\prime}}^{\lambda}\right) \circ\langle\mid v\rangle_{\lambda}=\langle\mid v\rangle_{\lambda \lambda^{\prime}} \circ\left(\mathrm{id} \otimes \iota_{\lambda, \lambda^{\prime}}^{\lambda}\right)$. Hence the equality $\rho_{\lambda} v_{\chi}=\rho_{\lambda^{\prime}} v_{\chi}$, which may be fully expanded as

$$
\left(\mathrm{id} \otimes \iota_{\lambda \lambda^{\prime}}^{\lambda}\right)\left(\mathrm{id} \otimes \iota_{\lambda}\right) \rho v_{\chi}=\left(\mathrm{id} \otimes \iota_{\lambda \lambda^{\prime}}^{\lambda^{\prime}}\right)\left(\mathrm{id} \otimes \iota_{\lambda^{\prime}}\right) \rho v_{\chi}
$$

implies that $\left\langle\rho_{\lambda} v_{\chi} \mid v\right\rangle=\left\langle\rho_{\lambda^{\prime}} v_{\chi} \mid v\right\rangle$, and by (8) this yields the wanted equality. The same way, using $\iota_{\lambda^{\prime} \lambda}^{\lambda}$ and $\iota_{\lambda^{\prime} \lambda}^{\lambda^{\prime}}$ in (9) this time, check the identity in another consecutive localization.

\section{Resolution of unity.}

A measure $\mu$ on a locally compact group $G$ is left-invariant if

$$
\int_{G} f\left(g g^{\prime}\right) d \mu\left(g^{\prime}\right)=\int_{G} f\left(g^{\prime}\right) d \mu\left(g^{\prime}\right)
$$

for all integrable functions $f$ on $G$ and for all $g \in G$. Here we may replace $f\left(g g^{\prime}\right)$ by $(\Delta f)\left(g \otimes g^{\prime}\right)$ where $\Delta$ is the comultiplication in a suitable topological Hopf algebra of functions on $G$. Evaluation at $g$ is a certain linear functional $h_{g}$ on that algebra. This motivates (25, 27])

DEFINITION 8. A left-invariant integral (= left Haar integral) on a Hopf algebra $H$ is a linear functional $\int$ on $H$ such that

$$
\left\langle h \otimes \int, \Delta(f)\right\rangle=\langle h, 1\rangle\left\langle\int, f\right\rangle, \quad \forall h \in H^{*} .
$$

A left Haar integral $\int$ is normalized if $\left\langle\int, 1\right\rangle=1$. 
Since linear functionals separate elements of $H$, the left invariance can be expressed as (dropping the evaluation brackets)

$$
\left(\mathrm{id} \otimes \int\right) \Delta(a)=\left(\int a\right) \cdot 1_{H}, \quad \forall a \in H .
$$

In other words, $1_{H} \int$ intertwines $H$ (as a right $H$-comodule with respect to the comultiplication) and its trivial subcomodule $\mathbb{C} \cdot 1_{H}$.

THEOREM 4. Let $\int$ be a left integral on a Hopf $*$-algebra H, and $(V, \rho,\langle\rangle$,$) a simple unitary right H$-comodule. Fix a vector $w \in V$. Define the operator $A: V \rightarrow V$ by

$$
A|v\rangle=\sum\left\langle w_{(0)} \mid v\right\rangle w_{(0)^{\prime}} \int w_{(1)}^{*} w_{(1)^{\prime}}
$$

Then $A$ is a scalar operator.

Proof. In the following, the primed Sweedler indices belong to another copy of the same variable, as in 25]. We compute directly

$$
\rho A v=\sum\left\langle w_{(0)} \mid v\right\rangle w_{(0)^{\prime}} \int w_{(1)}^{*} w_{(2)^{\prime}} \otimes w_{(1)^{\prime}}
$$

On the other hand,

$$
(A \otimes \mathrm{id}) \rho v=\sum\left\langle w_{(0)} \mid v_{(0)}\right\rangle w_{(0)^{\prime}} \int w_{(1)}^{*} w_{(1)^{\prime}} \otimes v_{(1)},
$$

what is by the left invariance of the integral equal to

$$
\sum\left\langle w_{(0)} \mid v_{(0)}\right\rangle w_{(0)^{\prime}} \int w_{(2)}^{*} w_{(2)^{\prime}} \otimes v_{(1)} w_{(1)}^{*} w_{(1)^{\prime}}
$$

and, by the coinvariance of the inner product,

$$
(A \otimes \mathrm{id}) \rho v=\sum\left\langle w_{(0)} \mid v\right\rangle w_{(0)^{\prime}} \int w_{(2)}^{*} w_{(2)^{\prime}} \otimes w_{(1)^{\prime}}
$$

We conclude that $\rho A v=(A \otimes \mathrm{id}) \rho v$. Hence the theorem follows from the Schur's lemma for comodules.

DEFINITION 9. $d \mu_{\lambda}(\chi):=\gamma_{\lambda}(\chi)\left(\gamma_{\lambda}(\chi)\right)^{*}$ in $\mathcal{E}_{\lambda}$.

THEOREM 5. Elements $\left|C_{\lambda}\right\rangle d \mu_{\lambda}(\chi)\left\langle C_{\lambda}\right|:=C_{\lambda} d \mu_{\lambda}(\chi) C_{\lambda}^{*}$ do not depend on $\lambda$ (agrees in all consecutive localization overlaps). Hence, by the globalization lemma, this family defines localized representatives of a unique expression $|C\rangle d \mu(\chi)\langle C|$ in $V \otimes \mathcal{G} \otimes V^{*}$. Taking a Haar integral in the tensor factor $\mathcal{G}$ yields a scalar operator $\alpha \cdot \mathrm{id}$ on $V$ (we identify "states" in $V \otimes V^{*}$ with operators). 
Remark: While $\left\langle C_{\lambda}\right|=C_{\lambda}^{*}$ should live in $\mathcal{G}_{\lambda} \otimes V^{*}$, we may define it only as a part of the expressions of the form $f^{*} C_{\lambda}^{*}:=\left(C_{\lambda} f\right)^{*}$ with $f \in \mathcal{G}$ such that $C_{\lambda} f \in V \otimes \iota_{\lambda}(\mathcal{G})$. Indeed, the involution $*$ is not defined on entire $\mathcal{G}_{\lambda}$, but only on $\mathcal{G}$, or if you wish, $\iota_{\lambda}(\mathcal{G})$.

Proof. Notice that in each local trivialization $\lambda$,

$$
\left|C_{\lambda}\right\rangle \gamma_{\lambda}(\chi)\left(\gamma_{\lambda}(\chi)\right)^{*}\left\langle C_{\lambda} \mid v\right\rangle=\sum\left\langle w_{(0)} \mid v\right\rangle w_{(0)^{\prime}} w_{(1)}^{*} w_{(1)^{\prime}}
$$

where on the LHS we assume that the pairing between $V^{*}$ and $V$ is assumed (applied) and on the RHS we assume appropriate localization. Recall that the product $C_{\lambda} \gamma_{\lambda}(\chi)$ does NOT depend on the localization. By (3) and Theorem 2 the RHS reads (no localizations this time) the element in $V \otimes \mathcal{G}$ to integrate. Hence by Theorem 4 we see that

$$
\int C_{\lambda} d \mu_{\lambda}(\chi) C_{\lambda}^{*}=\int\left|C_{\lambda}\right\rangle \gamma_{\lambda}(\chi)\left(\gamma_{\lambda}(\chi)\right)^{*}\left\langle C_{\lambda}\right|
$$

is a scalar operator.

In other words, if a family $\left\{C_{\lambda}\right\}_{\lambda}$ of coherent states exists, then the coherent states make a resolution of unity. This fact enables us to define an analogue of the Bargmann transform ([5]). To a vector $v \in V$ ( $V$ is physically a space describing some quantum numbers of the system; or a sector in a decomposition of such a space) we assign $\left\langle C_{\lambda} \mid v\right\rangle \in \mathcal{G}_{\lambda}^{\operatorname{co} \mathcal{B}}$. If $H$ is a linear operator on $V$, denote $H\left|C_{\lambda}\right\rangle:=(\mathrm{id} \otimes$ $H)\left|C_{\lambda}\right\rangle$. Suppose $\alpha \neq 0$ is the constant from Theorem [5. Then

$$
H|v\rangle=\alpha^{-1} \int H\left|C_{\lambda}\right\rangle d \mu_{\lambda}(\chi)\left\langle C_{\lambda} \mid v\right\rangle
$$

We then obtain (as in commutative case) a noncommutative version of a reproducing "integral" kernel on a Hilbert space, and equations involving $H$ (e.g. deformations of Schrödinger equation where $H$ is a Hamiltonian) can be written down in this coherent state representation.

\section{Comments on the quantum group case}

The simplest example concerns the coherent states for $\mathcal{G}=\mathcal{O}\left(S U_{q}(2)\right)$. We will mainly follow the notation and conventions of [22]. $\mathcal{O}\left(S L_{q}(2)\right)$ is a noncommutative Hopf algebra over $\mathbb{C}$ with 4 generators $a, b, c, d$, usually assembled in a matrix $T=\left(\begin{array}{ll}a & b \\ c & d\end{array}\right)$, with relations $a b=q b a$, $a c=q c a, b c=c b, b d=q d b, c d=q d c, a d-d a=\left(q-q^{-1}\right) b c$ and $\operatorname{det}_{\mathrm{q}} T:=a d-q b c=1 . \mathcal{O}\left(S U_{q}(2)\right)$ is a real form of $\mathcal{O}\left(S L_{q}(2)\right)$ 
determined by formulas $a^{*}=d, b^{*}=-q c, c^{*}=-q^{-1} b, d^{*}=a$. A vector space basis of $\mathcal{O}\left(S L_{q}(2)\right)$ is $\left\{a^{k} b^{r} c^{s}\right\}_{k>0, r, s>0} \cup\left\{b^{r} c^{s} d^{t}\right\}_{r, s, t>0}$. In particular, $\mathcal{O}\left(S L_{q}(2)\right)$ splits into a direct sum $\mathbb{C}[\zeta] \oplus \operatorname{compl}(\zeta)$ where $\mathbb{C}[\zeta]$ is the span of the basis elements of the form $(b c)^{r}$ and $\operatorname{compl}(\zeta)$ the span of the rest of basis. Notation $\mathbb{C}[\zeta]$ suggests that it is the algebra of polynomials in $\zeta=-q b c$, which will play major role below. $\mathcal{O}\left(S U_{q}(2)\right)$ posses a unique Haar functional $\int$, found by Woronowicz. With respect to the direct sum decomposition above, $\int$ is nontrivial only on $\mathbb{C}[\zeta]$ where it is given by formulas involving JACKSON's $q$-integral, or equivalently $(22])$

$$
\int \zeta^{r}=\frac{1-q^{-2}}{1-q^{-2(r+1)}}, \quad r=0,1,2, \ldots
$$

The lower quantum Borel subgroup $\mathcal{B}$ will be the quotient $\mathcal{O}\left(S L_{q}(2)\right) / I$, where $I$ is the 2 -sided ideal generated by $b . I$ is a Hopf ideal, hence $\mathcal{B}$ is a Hopf algebra. The quotient map $\pi: \mathcal{G} \rightarrow \mathcal{B}$ is datum (D1) from Sec. 5 , The images of generators are denoted $\lambda=\pi(a), \xi=\pi(c), \lambda^{-1}=\pi(d)$ and $\pi(b)=0$. Manin plane $\mathcal{O}\left(\mathbb{C}_{q}^{2}\right)$ is an algebra with two generators $x, y$ and a single relation $x y=q y x$. Elements of the form $x^{r} y^{s}$ form a basis of $\mathcal{O}\left(\mathbb{C}_{q}^{2}\right)$. The latter is a right $\mathcal{O}\left(S L_{q}(2)\right)$-comodule algebra via

$$
\rho\left(x^{r} y^{s}\right)=(x \otimes a+y \otimes c)^{r}(x \otimes b+y \otimes d)^{s} .
$$

$\mathcal{O}\left(\mathbb{C}_{q}^{2}\right)$ splits into the homogeneous components $V_{n}=\oplus_{r+s=n} \mathbb{C} x^{r} y^{s}$ of dimension $n+1$, which are irreducible and unitary. Our datum (D2) will be $\chi=\lambda^{-n}$ in $\mathcal{B}$, (D3) $V_{\chi}=V_{n}$, and (D4) will be the weight vector $v_{\chi}=y^{n}$. Datum (D5) is given by 1) two localizations $\mathcal{G}_{b}=\mathcal{G}\left[b^{-1}\right]$ and $\mathcal{G}_{d}=\mathcal{G}\left[d^{-1}\right]$ at Ore sets multiplicatively generated by $b$ and $d$ respectively; 2) comodule algebra maps $\gamma_{b}, \gamma_{d}$ obtained from the quantum Gauss decomposition. Let $u:=b d^{-1} \in \mathcal{G}_{d}$. It is easy to show ([36, 41]) that these localizations cover $\mathcal{G}$. Both localizations are $\rho_{\mathcal{B}}$-compatible, namely $\rho_{\mathcal{B}}$ extends by $\rho_{\mathcal{B}}\left(b^{-1}\right)=b^{-1} \otimes \lambda^{-1}$ and $\rho_{\mathcal{B}}\left(d^{-1}\right)=d^{-1} \otimes \lambda^{-1}$. The algebras of localized $\rho_{\mathcal{B}}$-coinvariants are given by $\mathcal{G}_{b}^{\text {co } \mathcal{B}}=\mathbb{C}[u]$ and $\mathcal{G}_{d}^{\text {co } \mathcal{B}}=\mathbb{C}\left[u^{\prime}\right]$ where $u=b d^{-1}$ and $u^{\prime}=d b^{-1}$. A unique ("Gauss") decomposition of matrix $T$ in the form $w U A$ where $w$ is a permutation matrix, $U$ upper triangular unidiagonal and $A$ lower triangular is possible in $\mathcal{G}_{b}$ with $w=\mathrm{id}$ and in $\mathcal{G}_{d}$ with $w=\left(\begin{array}{ll}1 & 0 \\ 0 & 1\end{array}\right)$. Map $\lambda \mapsto A_{1}^{1}, \lambda^{-1} \mapsto A_{2}^{2} \xi \mapsto A_{1}^{2}$ uniquely extend to a $\mathcal{B}$-comodule algebra map $\gamma_{d}: \mathcal{B} \rightarrow \mathcal{G}_{d}$, or to $\gamma_{b}: \mathcal{B} \rightarrow \mathcal{G}_{b}$ in the latter case. Explicitly $\gamma_{d}(\lambda)=a-b d^{-1} c, \gamma_{d}(\xi)=c, \gamma_{d}\left(\lambda^{-1}\right)=d, \gamma_{b}\left(\chi_{b}\right)=d^{n}$; $\gamma_{b}(\xi)=c-d b^{-1} a, \gamma_{b}(\lambda)=a, \gamma_{b}\left(\lambda^{-1}\right)=b$. Also $U_{2}^{1}=u$ in $\mathcal{G}_{d}$ and $U_{2}^{1}=u^{\prime}$ in $\mathcal{G}_{b}$. 
Analogously for general $n$, a cover of $\mathcal{O}\left(S L_{q}(n)\right)$ by $n ! \rho_{\mathcal{B}_{n}}$-compatible Ore localizations $S_{w}\left(w\right.$ in permutation group $\left.\Sigma_{n}\right)$ and $\mathcal{B}_{n}$-comodule algebra maps $\gamma_{w}: \mathcal{B}_{n} \rightarrow \mathcal{O}\left(S L_{q}(n)\right)\left[S_{w}^{-1}\right]$ is a highly nontrivial fact which we have shown elsewhere. It may be used to obtain the $\mathcal{O}\left(S U_{q}(n)\right)$ coherent states.

Using the $q$-binomial theorem, one obtains (in $V_{n} \otimes \mathcal{G}_{d}$ )

$$
\rho\left(y^{n}\right)=\sum_{i=0}^{n}\left[\begin{array}{c}
n \\
i
\end{array}\right]_{q^{-2}} q^{-\left(\begin{array}{c}
i \\
2
\end{array}\right)} x^{i} y^{n-i} \otimes u^{i} d^{n}
$$

Basis vectors $v_{i}^{n}=\sqrt{\left[\begin{array}{l}n \\ i\end{array}\right]_{q^{-2}}} x^{i} y^{n-i}$ are orthonormal. Thus

$$
C_{d}:=\sum_{i=0}^{n} q^{-\left(\begin{array}{c}
i \\
2
\end{array}\right)} \sqrt{\left[\begin{array}{c}
n \\
i
\end{array}\right]_{q^{-2}}} v_{i}^{n} \otimes u^{i}
$$

satisfies (7). Similar formula defines $C_{b}$ and the rest of requirements hold for these data. Thus

$$
A=\int_{S U_{q}(2)} \sum_{i, j=0}^{n} \sqrt{\left[\begin{array}{c}
n \\
i
\end{array}\right]_{q^{-2}}\left[\begin{array}{l}
n \\
j
\end{array}\right]_{q^{-2}}} q^{\left(\begin{array}{c}
i \\
2
\end{array}\right)+\left(\begin{array}{l}
j \\
2
\end{array}\right)} v_{i}^{n} \otimes\left(v_{j}^{n}\right)^{*} \otimes u^{i} d^{n}\left(u^{j} d^{n}\right)^{*}
$$

LEMMA 3.

$$
\int_{S U_{q}(2)} u^{i} d^{n}\left(u^{j} d^{n}\right)^{*}= \begin{cases}0, & i \neq j \\
{\left[\begin{array}{l}
n \\
i
\end{array}\right]_{q^{-2}}^{-1} q^{n} q^{2\left(\begin{array}{l}
i \\
2
\end{array}\right)[n+1]_{q}^{-1},}} & i=j\end{cases}
$$

Proof. $\left(u^{j} d^{n}\right)^{*}=q^{\left(\begin{array}{c}i \\ 2\end{array}\right)}\left(d^{*}\right)^{n-j}\left(b^{*}\right)^{j}=q^{\left(\begin{array}{c}i \\ 2\end{array}\right)}(-q)^{j} a^{n-j} c^{j}$. The identity

$$
d^{r} a^{r}=\left(1+q^{-1} b c\right)\left(1+q^{-3}(b c)^{2}\right) \ldots\left(1+q^{-2 n-1}(b c)^{r}\right)=\left(q^{-2} \zeta ; q^{-2}\right)_{r},
$$

implies $d^{n-i} a^{n-j}=d^{j-i}\left(q^{-2} \zeta ; q^{-2}\right)_{n-j}$ for $j \geq i$ and $\left(q^{-2} \zeta ; q^{-2}\right)_{n-j} a^{i-j}$ for $i<j$. Thus, for $j \geq i$,

$$
\begin{aligned}
u^{i} d^{n}\left(u^{j} d^{n}\right)^{*} & =q^{\left(\begin{array}{c}
i \\
2
\end{array}\right)+\left(\begin{array}{c}
j \\
2
\end{array}\right)}(-q)^{j} b^{i} d^{j-i}\left(q^{-2} \zeta ; q^{2}\right)_{n-j} c^{j} \\
& =q^{\left(\begin{array}{c}
i \\
2
\end{array}\right)+\left(\begin{array}{c}
j \\
2
\end{array}\right)}(-q)^{j} b^{i}\left(q^{-2} \zeta ; q^{2}\right)_{n-j} d^{j-i}
\end{aligned}
$$

what is for $j>i$ an element in $\operatorname{compl}(\zeta)$ hence it vanishes after integration, likewise an expression for $i<j$, and only the terms with $i=j$ survive. Then $u^{i} d^{n}\left(u^{i} d^{n}\right)^{*}=-q^{2\left(\begin{array}{c}i \\ 2\end{array}\right)} \zeta^{i}\left(q^{-2} \zeta ; q^{-2}\right)_{n-i}$, and using (52') in Chapter 4 of 22 one derives

$$
\int_{S U_{q}(2)} \zeta^{i}\left(q^{-2} \zeta ; q^{-2}\right)_{n-i}=\left[\begin{array}{c}
n \\
i
\end{array}\right]_{q^{-2}} q^{n}[n+1]_{q}^{-1},
$$


and the rest of the calculation is immediate.

Now

$$
\begin{gathered}
A=-\int_{S U_{q}(2)} \sum_{i=0}^{n}\left[\begin{array}{c}
n \\
i
\end{array}\right]_{q^{-2}}|i\rangle\langle i| \otimes \zeta^{i}\left(q^{-2} \zeta ; q^{-2}\right)_{n-i} \\
\int_{S U_{q}(2)} \sum_{i=0}^{n}\left[\begin{array}{c}
n \\
i
\end{array}\right]_{q^{-2}}|i\rangle\left\langle i\left|\otimes \zeta^{i}\left(q^{-2} \zeta ; q^{-2}\right)_{n-i}=[n+1]_{q}^{-1} q^{-n} \sum_{i=0}^{n}\right| i\right\rangle\langle i| .
\end{gathered}
$$

The sum on RHS is of course the unity. The fact that there was no additional factors depending on $i$ is the nontrivial property of coherent states (Theorem 5). There are many proposals for " $S U_{q}(2)$ coherent states" in literature (search e.g. MathSciNet) with similar (partly guessed) formulas with wrong $q$-factors and still having some "resolution of unity" formulas. The wrong factors are compensated by effectively changing the measure as well, for which there is no freedom as $S U_{q}(2)$ has only one invariant integral up to an overall constant!

In other words, $\alpha=q^{n}[n+1]_{q}^{-1}$ and the resolution of unity is

$$
I=q^{-n}[n+1]_{q} \int_{S U_{q}(2)}|C\rangle d \mu(\chi)\langle C| .
$$

Formula (10) boils down to an integral representation of RAMANUJAN's $q$-beta function (Theorem 10.3.1 in [1]; cf. also 4])

$$
\int_{0}^{1} x^{\alpha} \frac{(q x ; q)_{\infty}}{\left(q^{\beta} x ; q\right)_{\infty}} d_{q} x=\frac{\Gamma_{q}(\alpha) \Gamma_{q}(\beta)}{\Gamma_{q}(\alpha+\beta)} .
$$

If $\beta \geq 1$ is a positive integer, then the ratio in the integrand equals a polynomial in $q$ and $x$, namely, $(1-x)(1-q x) \ldots\left(1-q^{\beta-1} x\right)$. Like for the ordinary beta function, there is another integral representation involving $q$-integral from 0 to $\infty$ with a polynomial in the denominator. Namely, instead of the Haar integral over $\zeta=-q b c$ one effectively has a geometric integration over (deformed) 2-sphere with real coordinates $u=b d^{-1}$ and $\bar{u}$. However, in the denominator form, new $q$-factors appear depending on $q^{i}$. JURČO (15]) wrote a similar formula without extra $q^{i}$ factors, but both the "measure" and the coherent states are changed. Hence those "coherent states" do not satisfy the defining factorization property (17) and the measure is not the invariant one.

Computations of coherent states in selected local coordinates in concrete examples $\mathcal{O}\left(S U_{q}(n)\right)$ with $n=2,3$, appeared in [13, 15, 33, though without full geometric justification, and sometimes with nongeometric factors. Rudiments of another picture involving quantum group 
coherent states, related to geometric quantization and orbit method, are discussed in 42. Finally, a local picture (i.e. calculations in main Bruhat cell) of the coherent states for the case of compact forms of quantum groups of types $\mathrm{A}, \mathrm{B}, \mathrm{C}, \mathrm{D}$, which differs from but is related to ours, is in impressive work by JURČO and ŠŤOvíČEK ([16, 17]). Their family of coherent states, $\Gamma$ (cf. (5.1) in ([17])), live in $V \otimes \mathcal{G}$, i.e. generalize a map $G \rightarrow V$ rather than $G / B \rightarrow V$. They however calculate some expression in corresponding coordinates on a cell in homogeneous space, working in a big Zariski open cell (without rigorous justification for localization). Proposition 5.11 in ([17) is stating the factorization property (our formula (77) ) of their quantity $w_{\lambda}^{-1}\langle\Gamma, u\rangle$ which "belongs to some completion" and basically agrees with our coherent states. Their construction relies on structure properties of quantum groups, while our axiomatics allows a priori treatment of Hopf algebras of more general origin. Furthermore, our construction utilizes the globalization of the geometry on the quantum homogeneous space.

In commutative case, the elements of a family of coherent states form the projective orbit of the highest weight vector. The generators of $\mathcal{G}_{\lambda}^{\mathrm{co} \mathcal{B}}$ are the analogues of the local coordinates on a big open cell in the coset space, and the coherent vector $C_{\lambda}$ may be viewed as a parametrization of an open set in projective orbit by points in a coset space. In similar spirit, in the case of $\mathcal{O}\left(S L_{q}(3)\right)$, the reference 33. views $\mathcal{G}_{\lambda}^{\text {coB }}$ as an analogue of the (algebra of functions on) unipotent group parametrizes quantum orbit (though they note this algebra is not a bialgebra, unlike the classical case). Here we clarify that, as in the classical case, this should be understood as a parametrization of an open dense subset of orbit, the latter being a noncommutative space.

\section{Open question: minimal uncertainty}

It remains to study "covariant minimal uncertainty" properties of these coherent states. In the classical case, there is a quantity $\Delta(\mathbf{C})$ which is a sort of a "dispersion" of the Casimir element $\mathbf{C}$, and it is minimized on the coherent state orbit. We can show a quantum version by direct computation in one very simple example, but it remains to be studied in greater generality. An Ansatz for expression $\Delta\left(\mathbf{C}_{q}\right)$, which attains minimum at highest weight and in limit $q \rightarrow 1$ gives $\Delta(\mathbf{C})$, has been proposed for the case of the standard quantum groups possessing $R$-matrix in [9]. One may hope to reinterpret their expression in terms of braided Casimir element [12], and then place it into our geometrical context. In noncommutative case, individual coherent states are not defined, but the noncommutative 'family' of coherent states as a whole 
is still defined. Hence one may try to show that the average of the "dispersion" over the family of coherent states agrees with the minimal value over the whole representation space. In commutative case, this property is equivalent to saying that the minimum is achieved at "almost every" (in the measure sense, what forces "every" by continuity) point of the coherent state orbit.

Let me remark on possible strategies to obtain the minimization property. In the commutative case, the property can be traced to symplectic geometry. Up to a constant shift, and a negative multiple, the quantity $\Delta(\mathbf{C})$ equals the value of "the square of moment map" $\|\mu\|^{2}$, properly understood (43]). It is essential that the coherent state orbits are Kähler, hence symplectic. The square of moment map is extremal at symplectic orbits, hence the minimization of $\Delta\left(\mathbf{C}_{q}\right)$ ([43]). The noncommutative symplectic geometry developed by Kontsevich, GinzBurG and others ([11, 21] ) may suggest a path to extend the moment map argument to the noncommutative case.

A noncommutative infinitesimal neighborhood of a commutative scheme has been introduced by KAPRANOV ([18]). It is a very flexible setup for geometry with touch with direct calculations (FEYNMANMASLOV calculus). In the case of $q$-deformed groups one may hope to combine his approach with filtration arguments (with finite-dimensional geometry of graded pieces), and extend Morse-like arguments for study

of extremality.

\section{Acknowledgements}

I thank Prof. BAHA BALANTEKIN for suggesting the study of coherent states for quantum groups in a semesteral project in Fall 1996, and for his encouragement to try finding the "measure", what later resulted in $d \mu(\chi)$. I thank Prof. Joel W. RobBin for his patience, critique and many discussions on orbit method, coherent states and quantum groups at the University of Wisconsin-Madison. I thank the organizers of the 1999. summer workshop "Geometry and Physics" at Santa Barbara during which, in peace of my ITP office, Theorem 4 has been proved (and an imprecise version of Theorem 5) while supported in part by NSF under grant No. PHY99-07949. The project has been finished at the Indiana University, Bloomington, and the final version of the paper at the institutes Rudjer Bošković, Zagreb, and Max Planck, Bonn. 


\section{References}

1. G. E. Andrews, R. Askey, R. Roy, Special functions, Enc. Math. Appl. 71 Camb. Univ. Press (1999) xvi+664 pp. (Chap. 10: Introduction to q-series)

2. S. Twareque Ali, J. P. Antoine, J. P. Gazeau, U. A. Mueller, Coherent states and their generalizations : A mathematical overview, Rev. Math. Phys. vol.7, no.7 (1995), pp. 1013-1104.

3. J-P. Antoine, J-P. Gazeau, S. Twareque Ali, Coherent States, Wavelets and Their Generalizations, Grad. Texts in Contemp. Physics, Springer 2000.

4. R.Askey, Ramanujan's extensions of the gamma and beta functions, Amer. Math. Monthly 87 (1980), no. 5, pp. 346-359.

5. V.Bargmann, On a Hilbert space of analytic functions and an associated integral transform I, Comm. Pure and Applied Math. 14 (1961), pp. 187-214.

6. F. A. BerezIn, Quantization, Math. USSR-Izv. 38 (1974), no. 5, 1109-1165.

7. T. BRZEZIŃski, S. MAJID, Quantum group gauge theory on quantum spaces, Comm. Math. Phys. 157 (1993), no. 3, pp. 591-638.

8. A. Connes, Non-commutative geometry, Academic Press, New York 1994.

9. R. Delbourgo And R. B. Zhang, Minimal uncertainty states for quantum groups, J. Phys. A 30, L313-L316 (1997); arXiv:q-alg/9611021

10. V. G. DRINFEL'D, Quantum Groups, Proc. ICM-86, pp. 798-820.

11. V. GinzBurg, Non-commutative symplectic geometry, quiver varieties, and operads, math.QA/0005165

12. D. Gurevich, R. LeClercQ, P. Saponov, q-Index on braided noncommutative spheres Max Planck Institute Bonn preprint, MPI 2003 - 25.

13. L. K. Hadjitvanov, R. R. Paunov, I. T. Todorov, $\mathbf{U}_{q}$-covariant oscillators and vertex operators, J. Math. Phys. 33 (4), pp. 1379-1394, April 1992.

14. J. C. Jantzen, Representations of algebraic groups, Acad. Press 1987.

15. B. JURČO, On coherent states for the simplest quantum groups, Lett. Math. Phys., 21 (1991), pp. 51-58.

16. B. JURČO AND P. ŠTOVÍČEK, Quantum dressing orbits on quantum groups, Comm. Math. Phys. 152 (1993) pp. 97-126.

17. B. JURČO AND P. ŠTovíčEK, Coherent states for quantum compact groups, Comm. Math. Phys., 182 (1996), pp. 221-251; arXiv:hep-th/9403114

18. M. KAPRANOV, Noncommutative geometry based on commutator expansions, J. Reine Angew. Math. 505 (1998), pp. 73-118; arXiv:math.AG/9802041

19. V. V. KISIL, Two approaches to non-commutative geometry, in H. Begehr et al., Complex Methods for PDEs, pp. 219-248. Kluwer 1999; funct-an/9703001

20. V. V. KisiL, Integral representations and coherent states, Bulletin of the Belgian Mathematical Society, v. 2 (1995), No 5, pp. 529-540.

21. M. Kontsevich, Formal (non) commutative symplectic geometry, The Gel'fand Mathematical Seminars, 1990-1992, pp. 173-187, Birkhäuser 1993.

22. A. U. KLImyK, K. SChmüDGen, Quantum groups and their representations, Springer 1997.

23. W. LisieCKI, Coherent state representations. A survey., Reports on mathematical physics, vol. 35 (1995), No 2/3, pp. 317-358.

24. G. MACK AND V. Schomerus, Quasihopf quantum symmetry in quantum theory, Nucl. Phys. B370 (1992), pp. 185-230.

25. S. MAJID, Foundations of quantum group theory, Camb. Un. Press 1995, 2000.

26. YU. I. MANIN, Quantum groups and non-commutative geometry, CRM, Montreal 1988. 
27. S.Montgomery, Hopf algebras and their actions on rings, CBMS Regional Conference Series in Mathematics 82, AMS 1993.

28. A. M. Perelomov, Coherent states for arbitrary Lie groups, Comm. Math. Phys., 26 (1972), pp. 222-236; archived as arXiv:math-ph/0203002

29. A. M. Perelomov, Generalized coherent states and their applications, Texts and Monographs in Physics. Springer-Verlag, Berlin, 1986, xii+320 pp.

30. J. H. RAWnsley, Coherent states and Kähler manifolds, Quart. J. Math. Oxford (2), 28 (1977), pp. 403-415.

31. A. L. Rosenberg, Non-commutative affine semischemes and schemes, Seminar on supermanifolds 26, ed. D. Leites, Dept. Math., U. Stockholm 1988.

32. A.L. Rosenberg, Noncommutative schemes, Comp. Math. 112 (1998), 93-125.

33. H. SAzdjian, Y. S. Stanev, And I. T. Todorov, SU(3)-coherent state operators and invariant correlation functions and their quantum group counterparts, J. Math. Phys., 36 (1995), pp. 2030-2052.

34. H. J. Schneider, Lectures on Hopf algebras, Trabajos de Matemática 31, Universidad Nacional de Córdoba 1995, 58 pp.

35. H.-J. SCHNEIDER, Principal homogeneous spaces for arbitrary Hopf algebras, Israel J. Math. 72, (1990), nos.1-2, pp. 167-195.

36. Z. ŠKoDA, Coset spaces for quantum groups, Ph. D. Thesis, Univ. of WisconsinMadison, Jan. 2002 (available on request).

37. Z. ŠKODA, Localizations for construction of quantum coset spaces, in "Noncommutative geometry and quantum groups", P. M. Hajac, W. Pusz eds. Banach Center Publications vol.61, pp. 265-298, Warszawa 2003; arXiv:math.QA/0301090

38. Z. ŠKODA, Globalizing Hopf-Galois extensions, preliminary version.

39. Z. ŠKODA, Noncommutative localization in noncommutative geometry, arXiv:math.QA/0403276, $93 \mathrm{pp}$.

40. Z. ŠKODA, Quantum bundles using coactions and localization, in preparation.

41. Z. ŠKODA, Localized coinvariants I, II, preprints.

42. Y. SolBelman, Orbit method for the algebras of functions on quantum groups and coherent states I, Int. Math. Res. Notices 6 (1993), pp. 151-163.

43. M. SperA, On a generalized uncertainty principle, coherent states and the moment map, J. of Geometry and Physics 12 (1993) 165-182.

44. J. C. VARILLY, Hopf algebras in noncommutative geometry, lecture notes for the CIMPA Summer School in Villa de Leyva, arXiv:hep-th/0109077 